\title{
Research Paper \\ A Stereological Study on Colon Tissue Layers of Type 1 Diabetic Rats Following Thiamine and Lead Acetate Use
}

\author{
${ }^{*}$ Rahmat Allah Fatahian Dehkordi ${ }^{1}$ (i), Soren Nooraie ${ }^{1}$, Alborz Yadollahi ${ }^{1}$
}

1. Department of Basic Sciences, Faculty of Veterinary Medicine, University of Shahrekord, Shahrekord, Iran

\begin{tabular}{|c|c|}
\hline $\begin{array}{l}\text { Use your device to scan } \\
\text { and read the article online }\end{array}$ & Cttation: Fatahian Dehkordi R, Nooraie S, Yadollahi A. [A Stereological Study on Colon Tissue Layers of Type 1 Diabet- \\
\hline arita & $\begin{array}{l}\text { ic Rats Following Thiamine and Lead Acetate Use (Persian)]. Journal of Arak University of Medical Sciences (JAMS). 2021; } \\
\text { 24(1):36-49. https://doi.org/10.32598/JAMS.24.1.4927.2 }\end{array}$ \\
\hline 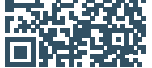 & doil https://doi.org/10.32598/JAMS.24.1.4927.2 \\
\hline
\end{tabular}

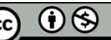

Article Info:

Received: 28 Nov 2019 Accepted: 05 Oct 2020 Available Online: 01 April 2021

Keywords:

Thiamine, Stereology, Diabetes, Rats, Colon

\section{A B STRACT}

Background and Aim Diabetes is a well-known disease with such complications, as retinopathy, nephropathy, and gastropathy. This study aimed to investigate the effects of thiamine and lead acetate on the colon of induced-alloxan diabetic rats; the effects of which become obvious in the treatment or reduction of tissue complications caused by diabetes.

Methods \& Materials In this study, 63 rats weighing 200 g were divided into 9 groups, as follows: 1) Group of diabetes+pb acetate 200 ppm; 2) Group of thiamin+pb acetate 200 ppm; 3) Group of thiamine+pb acetate 1000 ppm; 4) Group of diabetes+thiamine+Pb acetate 1000 ppm; 5) Diabetes group; 6) Group of diabetes+thiamine; 7) Group of diabetes+thiamine+acetate 200 ppm; 8) Group of diabetes+pb acetate 1000 ppm, and 9) the control group. After 20 days, the study samples were removed from the abdominal cavity and the slides were prepared by routine tissue method. Then, the slides were evaluated for stereological and histomorphometric studies.

Ethical Considerations This study was approved by the Faculty of Veterinary Medicine, Shahrekord University (Code: GRN1M1903). Moreover, all methods used in the present study, including facilitation, were conducted per the ethical principles of animal restraint.

Results The mean thickness of mucosa-sub-mucosa suggested significant differences in groups 6 and 7 , compared to other treatment groups. There was a significant difference in the thickness of the muscle layer between the control and all treatment groups except for groups 2, 6, and 7. There was no significant difference in the mean thickness of advantia layer in groups 1, 7, and 8, and the control group. The obtained results also indicated a significant difference concerning different layers of colon tissue between group 1 and controls.

Conclusion Based on the present research results, thiamine presented enhancing effects on muscle layer thickness and adventitia layer thickness. Furthermore, the area of the mucosal layer was not affected by the improving effects of thiamine.

\section{Extended Abstract}

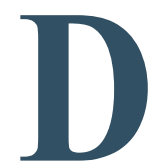

\section{Introduction}

iabetes is a well-known disease, i.e., generally caused by the disorder of insulin hor- mone metabolism, the disorder of hormone production, or the disruption of the production of membrane cell surface receptors on its target cells [4]. Diabetes has such complications as, retinopathy, nephropathy, and the involvement of the main peripheral arteries; it presents gastrointestinal motility disorders [5]. Thiamine is the first known B vitamin

\section{* Corresponding Author:}

Rahmat Allah Fatahian Dehkordi, PhD.

Address: Department of Basic Sciences, Faculty of Veterinary Medicine, University of Shahrekord, Shahrekord, Iran.

Tel: +98 (38) 32324427

E-mail: fatahian_1349@yahoo.com 
in energy supply, fat metabolism, and nucleotides. Besides, it plays an essential role as a coenzyme, especially in the formation and development of brain cells [8]. The accumulation of lead in the body can have destructive effects on the blood, bone marrow, kidney, and gastrointestinal blood cells [12]. In a report (Leff 2018), the injection of lead acetate in male mice with diabetes mellitus increased fasting blood glucose levels [15]. This study was designed to investigate the effects of thiamine and lead acetate on the colon of diabetic rats.

\section{Materials and Methods}

In all stages of this research, the ethical principles of working with laboratory animals in the laboratory of Shahrekord University were considered. This study was approved by the Research Ethics Committee of Shahrekord Veterinary School. In total, 63 rats with an average weight of 220-200 $\mathrm{g}$ were prepared from the animal house of Shahrekord University.

The examined rats were classified into 9 groups, as follows:

1) Diabetes+acetate group $200 \mathrm{ppm}$;

2) Thiamine+acetate group $200 \mathrm{ppm}$;

3) Thiamine group+lead acetate $1000 \mathrm{ppm}$;

4) Diabetes+thiamine+lead acetate 1000 ppm group;

5) Diabetes group;

6) Diabetes+thiamine group;

7) Diabetes+thiamine+acetate group $200 \mathrm{ppm}$;

8) Diabetes+acetate group $1000 \mathrm{ppm}$;

9) Control group.

After 20 days of treatment, the explored rats were anesthetized with ether anesthetic and facilitated by human methods. The abdominal cavity was opened and the large intestine came out of the cavity. Different layers of colon tissue, including mucosa, submucosa, muscle, and adventitia were examined under a microscope for histology and histometric. For stereological evaluation, using point grade, the area of different wall layers of the colonic tissue structure was examined.

\section{Results}

The morphometric findings revealed that the mean thickness of the mucosal-submucosal layer was the highest in treatment group 6 (diabetes+thiamine) and the lowest in group 7 (diabetes+thiamine+acetate $200 \mathrm{ppm}$ ) (Figures 1 A, B \& C). These results also indicated that these differences were significant in groups 6 and 7 , compared to other treatment groups and the controls, except for group 5 (diabetic group) concerning analysis at $\mathrm{P}<0.05$. Morphometric results revealed a significant difference in the mean thickness of the smooth muscle layer of the colon wall in treatment groups 1 to 8 , except for group 2 (thiamine $+200 \mathrm{ppm}$ lead acetate), compared to the control group $(\mathrm{P}<0.05)$. The obtained results presented no significant difference between the mean thickness of the muscle layer of group 2 and the control group $(\mathrm{P}<0.05)$. The average area of the mucosalsubmucosal layer data suggested that in groups 1 and 8 , there was a significant difference, compared to the control group $(\mathrm{P}<0.05)$; in both of them, a decrease was observed in this area. The results of the area parameter study in the muscle layer of the colon indicated that only in group 1, there was a significant difference, compared to the control group $(\mathrm{P}<0.05)$. Besides, this difference was in the direction of increasing the area.

Examining serous layer surface presented no significant difference, compared to the control group $(\mathrm{P}<0.05)$. There was only a significant difference between the groups of diabetes+lead 200 acetate and thiamine+lead 200 acetates in this respect.

\section{Discussion and Conclusion}

The present study investigated the histomorphometric changes of different layers of the colon in diabetic rats. As the present study revealed, the effects of thiamine and lead acetate in two different doses were well evident on the colon wall layers. The obtained data highlighted a significant decrease in the thickness of the mucosal-submucosal layer of groups 1, 7, and 8 (diabetes + acetate $1000 \& 200$ groups $\&$ diabetes + thiamine + lead acetate 200 groups), compared to the control group; this decreasing trend well reflects the damaging effects of lead acetate on the thickness of the mucosal-submucosal layer. The results of some studies suggested that free radicals are the main cause of many disorders in diabetes [21]. The collected results indicated that the thickness of the muscle layer, including decreasing changes in group 5 (diabetes) compared to the controls. However, Nowak et al. (1995) found that, in contrast to the data of the present study, induced experimental diabetes increased the thickness of the muscle layer [27]. Surface findings from the study of colonic tissue stereology 


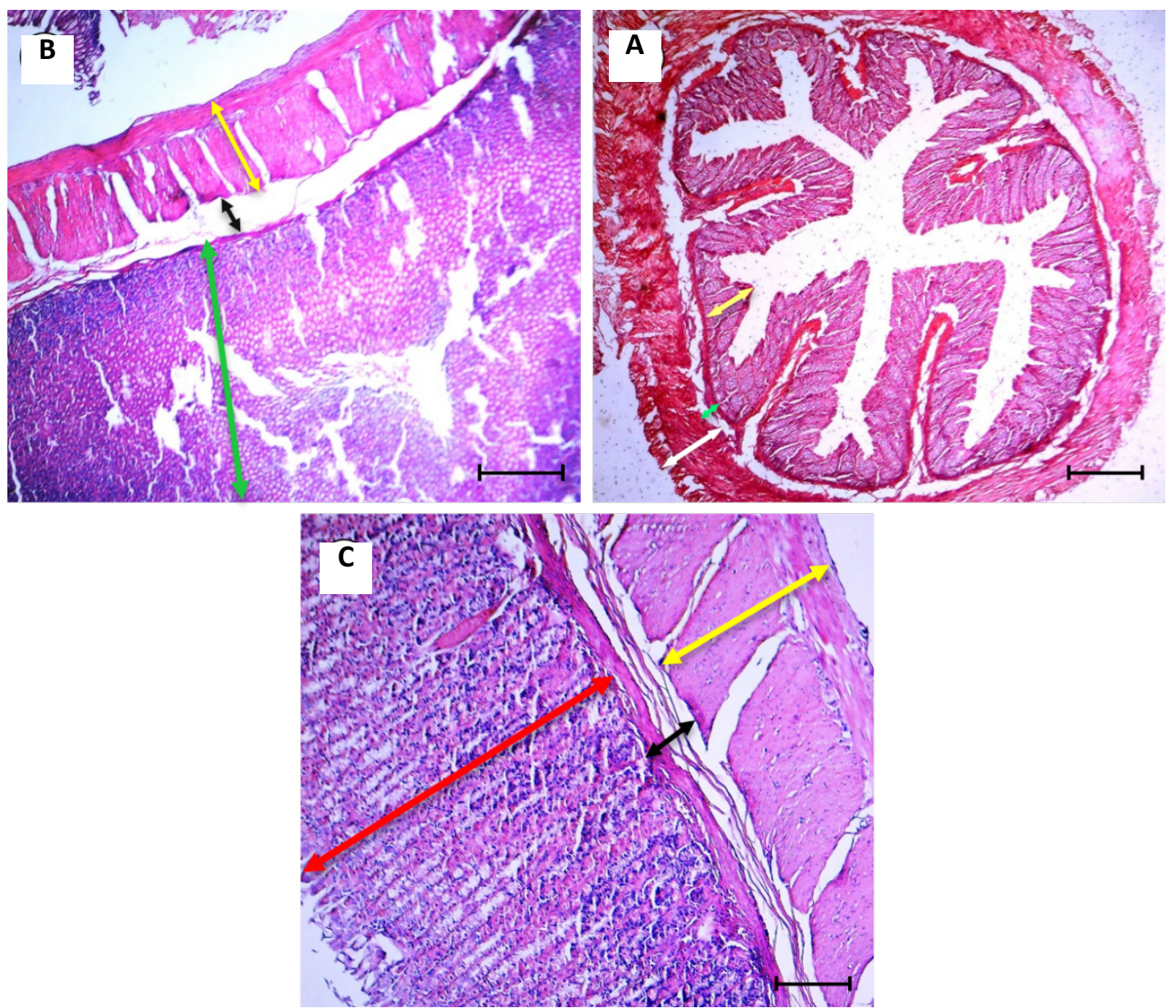

5 . Jing University of Medical Sciences

Figure 1. Representative photomicrograph showing quantitative histomorphometric changes of colon tissues from rats

Figure A. The micrograph of colonic tissue in the control group

Note the regular tissue structure of the various layers of the colon wall. Mucous layer (yellow double-sided arrow) with submucosal layer (green double-sided arrow) and muscle layer (white double-sided arrow) (hematoxylin and eosin staining; Bar: 50 $\mu \mathrm{m}$ ).

Figure B. The micrograph of colon tissue in the diabetic group

Note the disjointed tissue structure of the various layers of the colon wall. The mucosal layer (green double-sided arrow) is well visible along with the submucosal layer (black double-sided arrow) and the muscle layer (yellow double-sided arrow) (hematoxylin and eosin staining; Bar: $15 \mu \mathrm{m})$.

Figure C. Colon tissue micrograph in the group of diabetes + thiamine + acetate $1000 \mathrm{ppm}$

Note the tissue structure of the different layers of the colon wall. The mucosal layer (red bilateral arrow) is visible along with the submucosal layer (black bilateral arrow) and the muscular layer (yellow bilateral arrow) (hematoxylin and eosin staining; Bar: $15 \mu \mathrm{m}$ ).

presented that in group 1, the average area of different tissue layers was significantly different from the control group. These changes signified the effects of diabetes and lead acetate on colon tissue in the direction of increasing (mucosal layer area-submucosal) and decreasing (muscle layer area and adventitia). The results of another study revealed that diabetes caused a peripheral and longitudinal enlargement of the colon wall [29]. Considering the induction of experimental diabetes and subsequent administration of thiamine in the rats of the treatment group, thiamine, in some of the studied groups, has improved effects on muscle layer thickness and adventitia layer thickness. However, the mean area of the mucosal layer was not affected by the improving effects of thiamine. 


\section{Ethical Considerations}

\section{Compliance with ethical guidelines}

This study was approved by the ethics code GRN1M1903 in the Faculty of Veterinary Medicine of Shahrekord University. Furthermore, all the methods used, including facilitation, were performed per the ethical principles of animal restraint.

\section{Funding}

The project was supported by Faculty of Veterinary Medicine, Vice Chancellor for Research, Shahrekord University.

\section{Authors' contributions}

Conceptualization: Rahmat Allah Fatahian, Soren Nouraei; Research: Rahm Atollah Fatahian, Alborz Yadalahi; Editors: Soren Nouraei, Alborz Yadalahi, Rahmat Allah Fatahian.

\section{Conflicts of interest}

The authors declared no conflicts of interest.

\section{Acknowledgements}

The authors want to thank all staff of the Physico-Pharmacology and Histo-Pathology Laboratories, as well as the esteemed Vice Chancellor for Research of Shahrekord University and Faculty. 
This Page Intentionally Left Blank 


\section{بررسى استريولوريك لايههاى بافتى كولون موشهاى ديابتى نوع ا به دنبال مصرف ثيامين و}

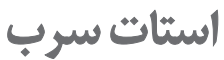

"رحمتالله فتاحيان دهكردى' :ه، سورن نورايى'، البرز يدالهى'

1. كروه علوم بايه، دانشكده داميزشكى، دائشكاه شهركرد، شهركرد، ايران.

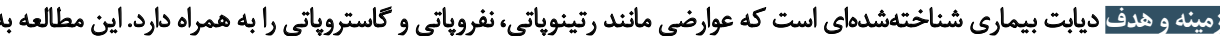

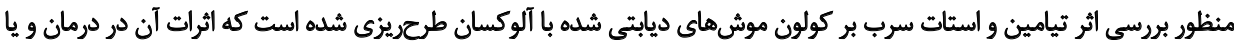

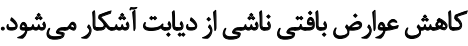

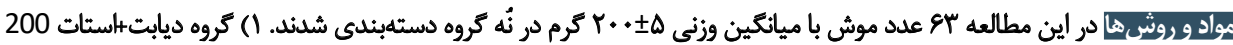

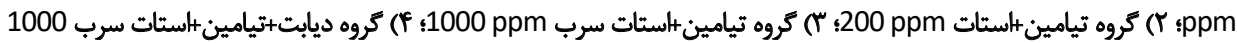

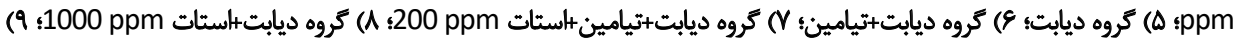

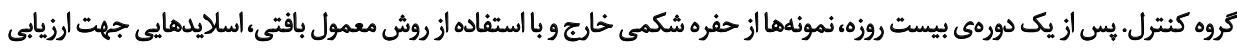

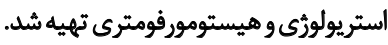

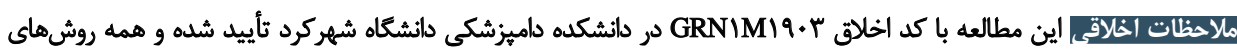

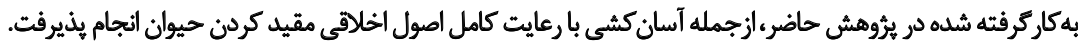

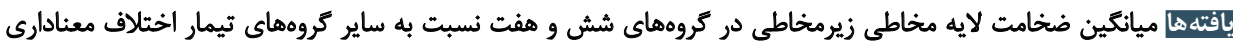

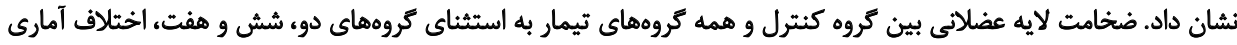

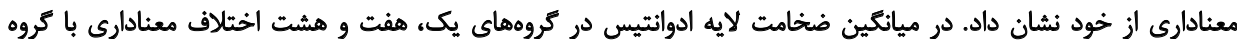

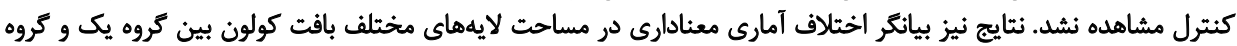

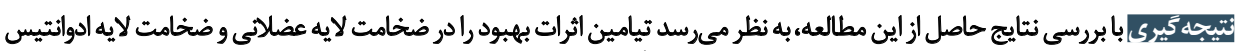

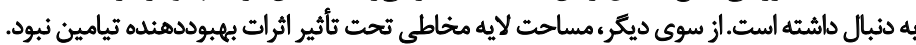

اين بيمارى در دئيا بيمارى شناختهشداي استكه به طور

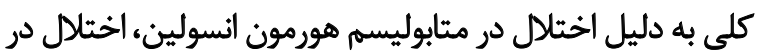

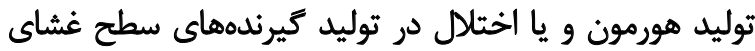

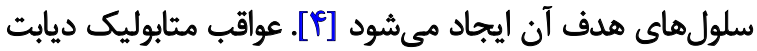

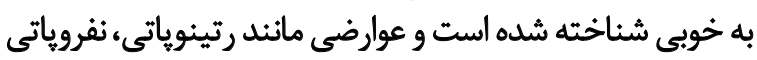

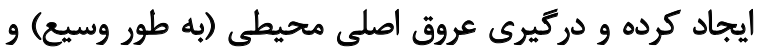

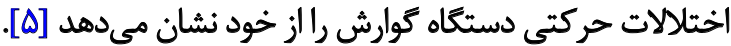
ازجمله عوارضى كه ديابت در دستكاه كوارشى ايجاد مي كند

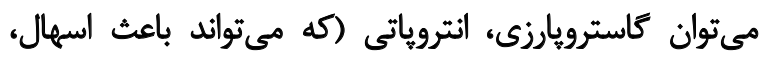
بىاختيارى مدفوع و يبوست شود) و كبد جرب غيرنيان الكلى ران نام
مقدمه

اختلال شايعى كه به طور جشم گير در بيشتر كشورها، ازجمله

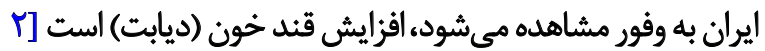

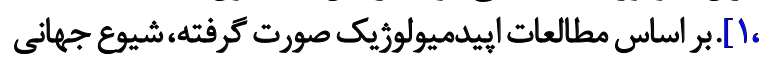

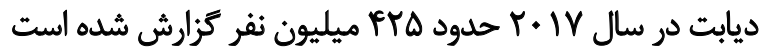

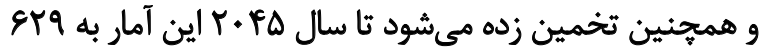

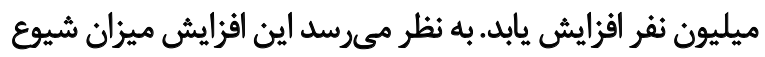

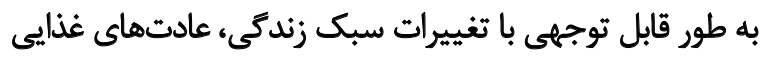

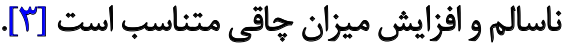

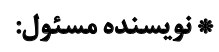

دكتر رحمثالله فتاحيان دهكردى : نويند

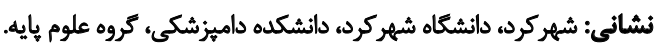
تلفن: يست الكترونيكي: fatahian_1349@yahoo.com 


\section{بافتشناسى و استريولوزى بود. \\ مواد وروش \\ ميوائات و شرأيط نتكهاري}

در تمام مراحل اين تحقيق، اصول اخلاقى كار با حيوانات

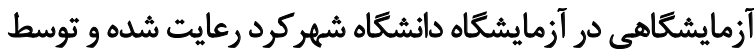

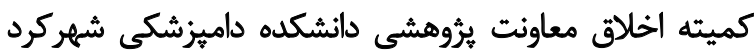
مورد تأييد قرار ترفت.

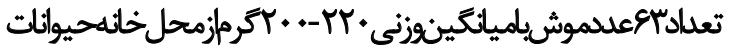

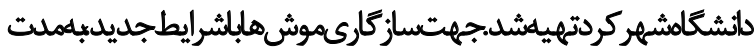

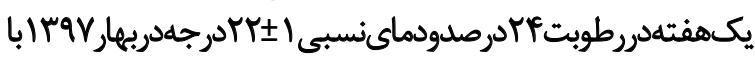
سيكل روشنايى تاريكى دوازده ساعته نكلهدارى شدند.

به منظور ايجاد محيط مناسب براى موشها، كف قفسها با

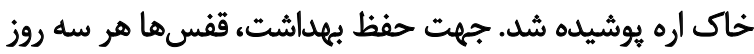

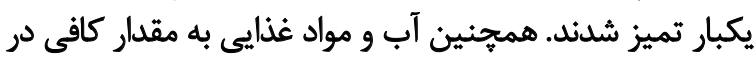
اختيار حيونات قرار ترفت.

كوروهئمي

بس از سازكارى موشها با محيط جديد، حيوانات به صورت

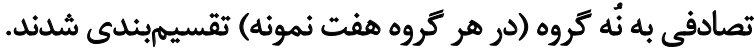

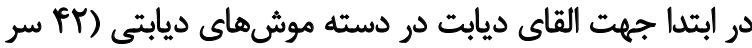

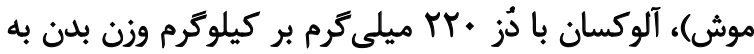

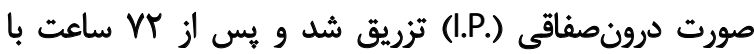

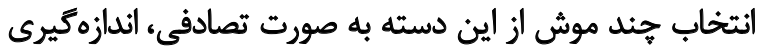
قند خون آنها در شرايط ناشتا جهت تأييد ديابتى شدن مون موشها

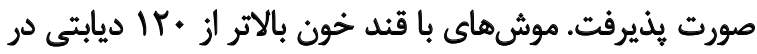

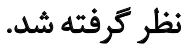

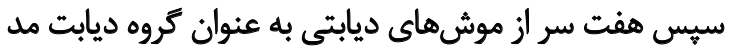

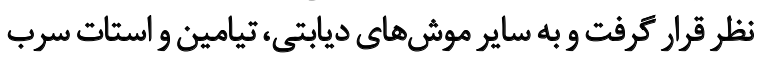

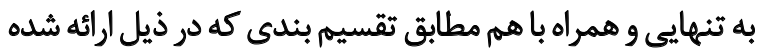

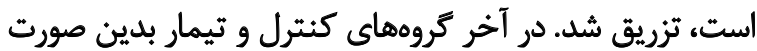
قابل جمعبندى است:

مروه ا: به عنوان كروه ديابت (آلوكسان بادُز همراه با استات سرب (200 ppm) در نظر كرفته شد.

كروه ז:اين كروه به عنوان كروه تيامين (mg/kg 70) همراه با

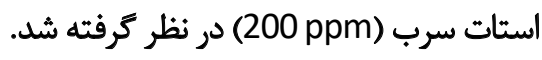

كروه "ا: به عنوان موشهاي گروه تيامين (mg/kg 70) همراه

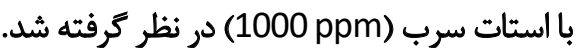

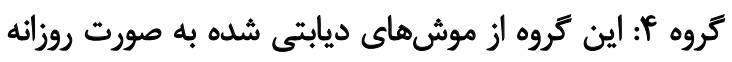

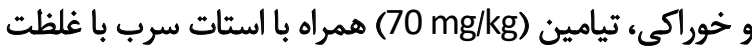

برد [\&]. ارتباط بين شرايط ميكروبى روده و هموستاز بدن و و

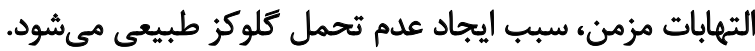

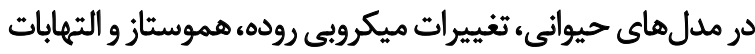

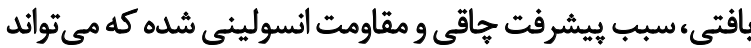

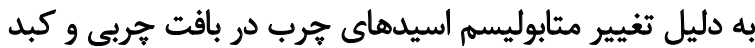

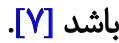

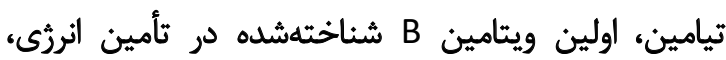

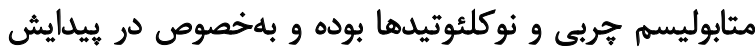

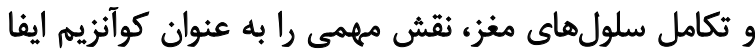

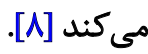

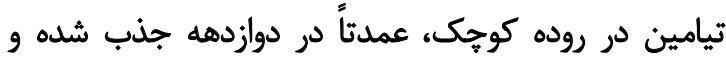

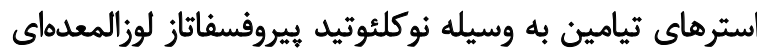

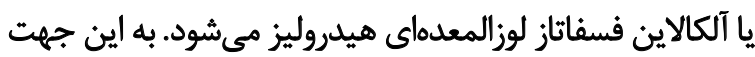

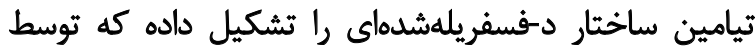

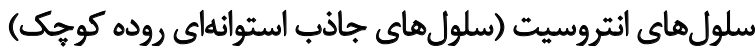

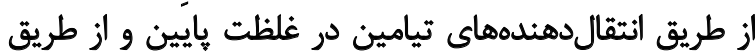

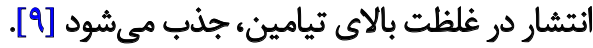
ارتباط بين ثيامين و ديابت در بيشتر مقالات و نشريات

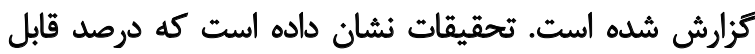

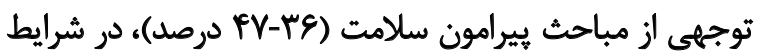

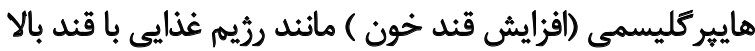

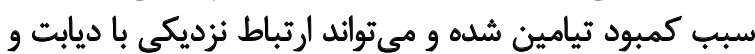

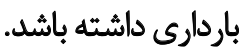

در مطالعهاى كه توسط لانت و همكاران انجام شد ذخاير

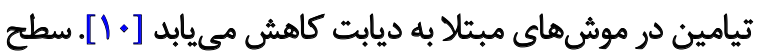

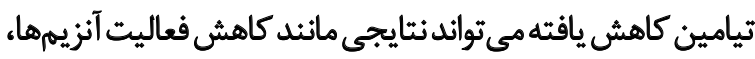

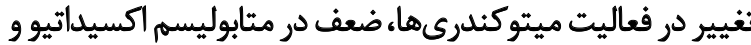

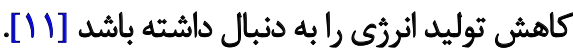
تجمع سرب در بدن مى تواند آثار مخربى در خون، سلولهاي

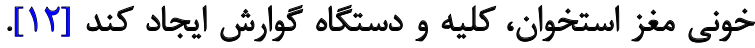

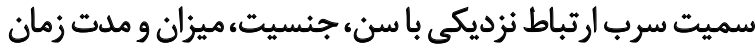

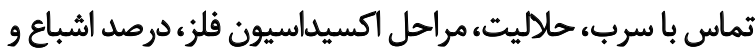

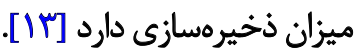
سرب مىتواند از طريق جذب يوستى يا تنفس (دم) به وسيله

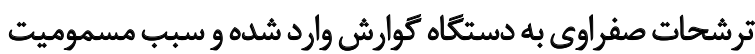

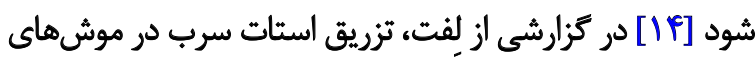

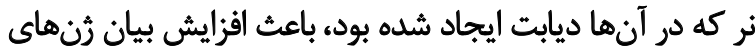

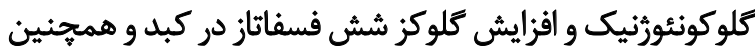

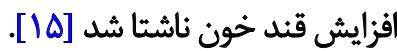
هدف از يروهش حاضر، بررسى اثر تيامين بر موشهاى دئ ديابتى

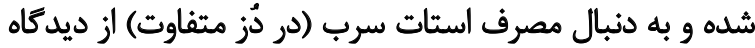


مشخصشدهاست.تصاويرشمارها اتآبيان گرساختاربافتى كولوندر

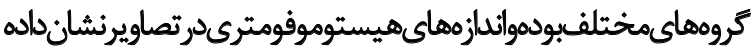

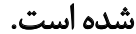

در گروه كنترل، ساختار بافتى منظم كولون، شامل لايههاى

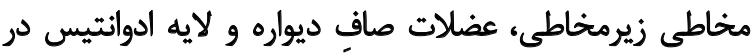
نمونهاى تهيهشده، مشاهده شد (تصوير شماره ل). يرزها در داخل ساختار يادشده به شكل منظم خود ديده

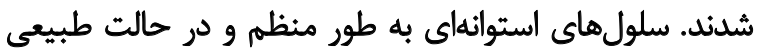

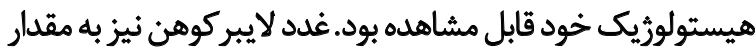

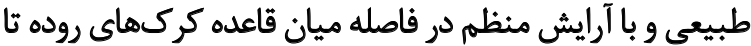
ماهيحه مخاطى قرار داشتند.

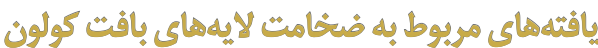

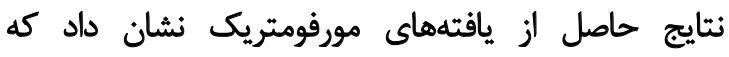
ميانكين ضخامت لايه مخاطى زيرمخاطى دئى در كرون

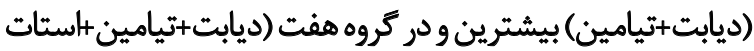

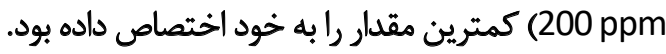
اين نتايج نيز نشان داد كه اين اختلافات در كروههاى شش و و

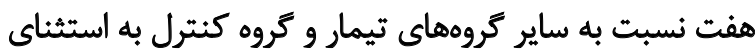

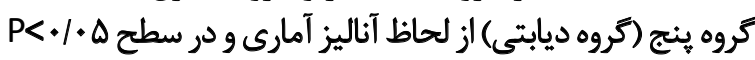

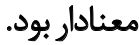

نتايج مورفومتريك نشان داد كه اختلاف آمارى معنادارى در

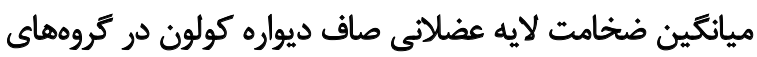

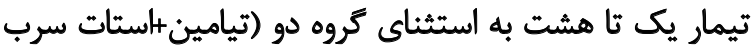
200ppm نسبت به كروه كنترل وجود دارد (ه + (P> (P). يافتهها مشخص كرد كه هيجّونه اختلاف آمارى معنادارى

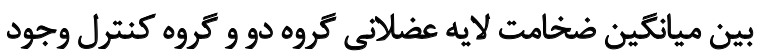

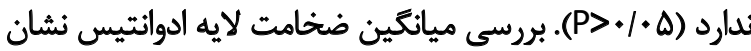

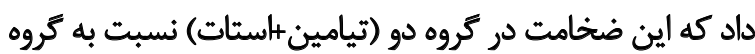

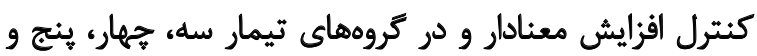

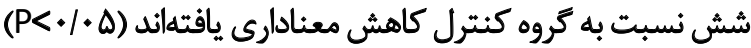

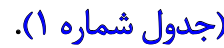

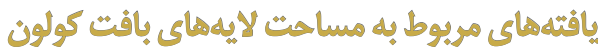

يافتههاى بهدستآمده از آناليز آمارى ميانكين مساحت لايه

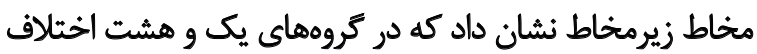

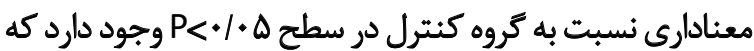
در هر دوى آنها كاهش مساحت مشاهرو كنترل در سطح شد. نتايج حاصل از بررسى يارامتر مساحت در لايه عضلاتى كولون

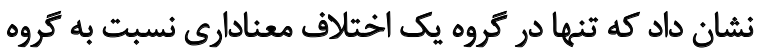

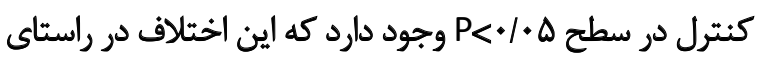

1000 ppm

كروه ه: به عنوان موشهاى ديابتى در نظر كرفته شد. كروه 8: موشهاى ديابتى شدهاي هستئد كه تيامين را با دُز إن 70 mg/kg صورت داخل صفاقى دريافت كردند.

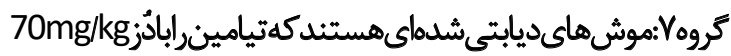
بهوروت داخل صفاقىواستاتسربرابراباغلظت 200ppmبهصورت

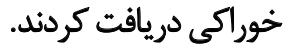

كروه ^: موشهايى هستند كه ديابتى بوده و استات سرب خوراكى را با غلظت موشمايى هرو 1000 مصرف كردند.

كروه 9: هوشهايى هستند كه ديابتى نشده و به عنوان كروه

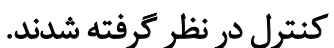

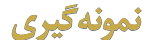

يّ إز يك دوره تيمار بيست روزه، موشها توسط داروى

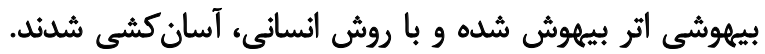

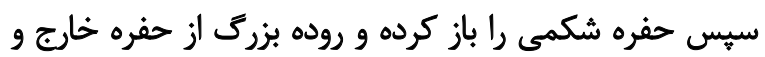

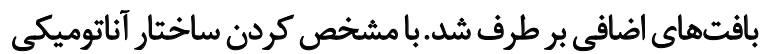

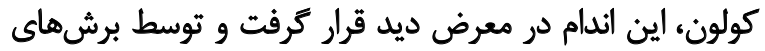

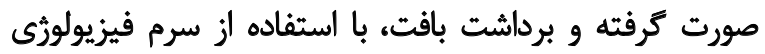

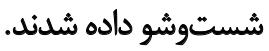

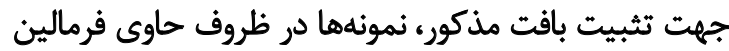

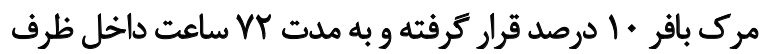

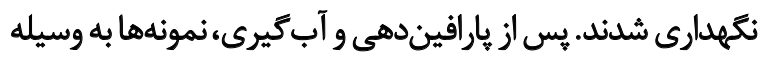

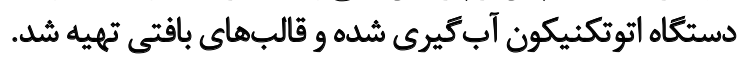

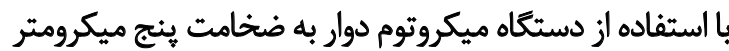

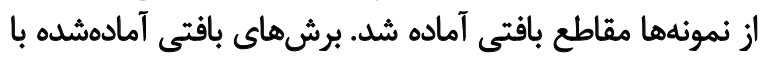

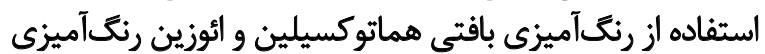

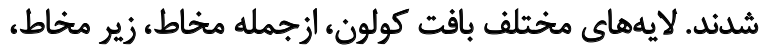

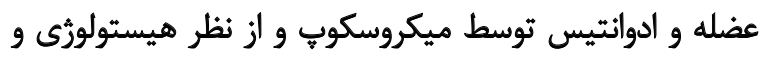

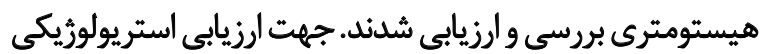

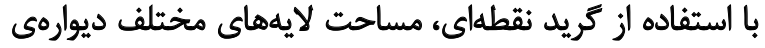

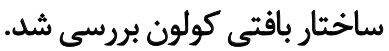

ياقثلهها

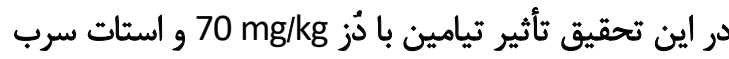

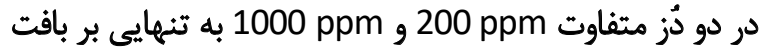

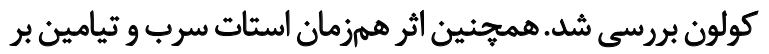
موشهاى ديابتى ارزيابى شد.

نتايجاينمطالعهبريايهاندازمخيرىضخامت لايههائمختلفبافتئي

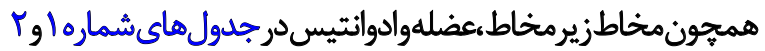


جدول ا. مقايسه ميانكين ضخامت لايهماي مختلف مخاط زيرمخاط، عضله و ادوانتيس در كروهانى مطالعاتى كولون موشهاي نر بالغ بعد از بيست روز تيمار

\begin{tabular}{|c|c|c|c|c|c|}
\hline \multicolumn{3}{|c|}{ ميانكين土|نحراف معيار } & \multirow{2}{*}{$\mathbf{P}$} & \multirow{2}{*}{ 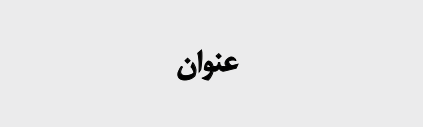 } & \multirow{2}{*}{ 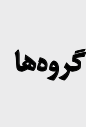 } \\
\hline أدواتثيس (ر) & عضله ( ع) & مخاط زير مخاط ( & & & \\
\hline VNDE. $/ T$ & WA \pm$\}^{*}$ & $r e / R \pm r / \Lambda^{*}$ & $<+1 \cdot \Delta$ & 200 ppm ديابت+استاث سرب & كرو اه \\
\hline$\vee / \Delta f \pm \cdot / e^{*}$ & $\operatorname{rep} \Delta \pm \cdot / 9$ & efiftr & $<\cdot 1 \cdot \Delta$ & 200 ppm تيامين +استات سرب & $r \Delta \log ^{5}$ \\
\hline$f / r \pm \cdot / e *$ & $\mid r / g \pm r$ & $r \cdot / e \pm f / r$ & $<\cdot / \cdot \Delta$ & 1000 ppm تيامين+استات سرب & $r \Delta g$ \\
\hline$r / \Lambda \Delta \pm \cdot / r^{*}$ & $10 \pm 1^{*}$ & $r \varepsilon / \& \pm Y / R$ & $<+1 \cdot \Delta$ & 1000 ppm ديابت+تيامين+|(سثات سرب & $\log 5$ \\
\hline$\Delta / r \gamma \pm \cdot / r^{*}$ & $W A \pm \mathbb{1} A^{*}$ & $r \Delta \pm r / \varphi$ & $<+1 \cdot \Delta$ & ديابت & $\Delta \Delta, \xi$ \\
\hline$\varphi / \varphi \Delta \pm . / \%$ & $19 / r \pm 1 / 1^{*}$ & $\Delta \& / \Delta \pm \psi / \varphi^{*}$ & $<+1 \cdot \Delta$ & ديابت+تيامين & $\operatorname{sog} 5$ \\
\hline$V / A \pm \cdot / 8$ & $r+/ r \pm 1 / r^{*}$ & $r \cdot \pm r / \ell^{*}$ & $<+1+\Delta$ & 200 ppm ديابت+تيامين+استات سرب & $V \circ g$ \\
\hline $8 / 1 \pm \cdot 18$ & $W F \pm 1 / 1^{*}$ & $r \cdot / r \pm r / q^{*}$ & $<+1 \cdot \Delta$ & 1000 ppm ديابت+استات سرب & 10 \\
\hline$V / \& \pm \cdot / T$ & $r N T \pm T / A$ & $f \cdot 1 \& \pm T / V$ & $<\cdot 1 \cdot \Delta$ & 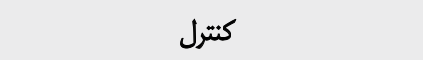 & 9 \\
\hline
\end{tabular}

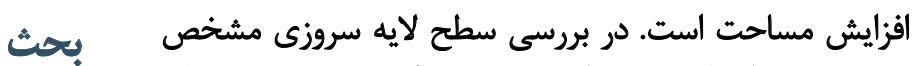

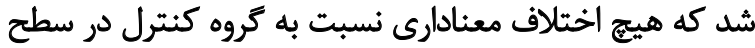

مطالعه حاضر تغييرات هيستومورفومتريك لايههاى مختلف

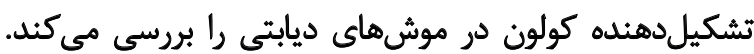

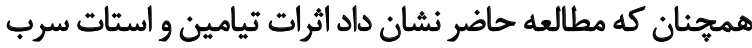

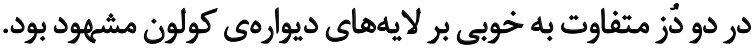

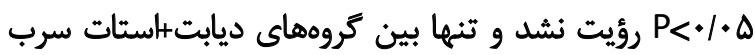

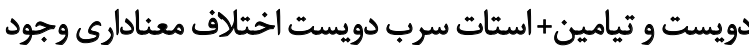
دارد (جدول شمارمى T).

جدول r. مقايسه ميانكين مساحت لايههاى مختلف مخاط زيرمخاط، عضله و ادوانتيس در تروههاى مطالعاتى كولون موشهاى نر بالغ بعد از بيست روز تيمار

\begin{tabular}{|c|c|c|c|c|c|}
\hline \multicolumn{3}{|c|}{ ميانكين |انحراف معيار } & \multirow{2}{*}{$\mathbf{P}$} & \multirow{2}{*}{ عنوان } & \multirow{2}{*}{ كروهاها } \\
\hline 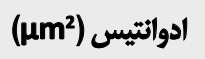 & عضله ( & مخاط زيرمخاط ( & & & \\
\hline$\cdot / r \cdot \pm \cdot / \cdot 1$ & $\cdot / R \pm \cdot / 10^{*}$ & $. / r \checkmark \pm .1 .8^{*}$ & $<+/ * \Delta$ & 200 ppm ديابت+استات سرب & كروها \\
\hline$. / r \pm . / \cdot r$ & $\cdot / r q \pm \cdot / * r$ & $\cdot / \Delta \Delta \pm=/ 1$ & $<\cdot / \cdot \Delta$ & 200 ppm تيامين+استات سرب & r \\
\hline$. / / \vee \pm . / . \mu$ & $. / 19 \pm+/ .0$ & $. / 9 Y \pm . / . V$ & $<\cdot / \cdot \Delta$ & 1000 ppm تيامين+استات سرب & كروه \\
\hline$. / 19 \pm . / .1$ & $. / 19 \pm .1 .8$ & $\cdot / \Delta q \pm \cdot / \bullet \Delta$ & $<\cdot 1 \cdot \Delta$ & 1000 ppm ديابت+تيامين+|ستات سرب & كروه \\
\hline$. / r \pm . / . r$ & $. / 19 \pm . / \circ \Delta$ & $. / 9 r \pm .1 .8$ & $<\cdot 1 . \Delta$ & ديابت & كروه \\
\hline$\cdot / r \cdot \pm \cdot / \cdot \varphi$ & $\cdot / M T \pm \cdot / * F$ & $\cdot / \Delta F \pm \cdot / * r$ & $<+1 \cdot \Delta$ & ديابت+تيامين & كروه \\
\hline$. / / \vee \pm . / . \varphi$ & $. / r q \pm . / .9$ & $\cdot / \Delta Y \pm \cdot / \cdot q$ & $<\cdot / \cdot \Delta$ & 200 ppm ديابت+ثيامين+استات سرب & 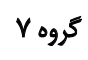 \\
\hline$. / Y Y \pm \cdot / \cdot r$ & $\cdot / r \cdot \pm \cdot / \cdot r$ & $\cdot|\Delta| \pm \cdot / \cdot 1$ & $<\cdot / \cdot \Delta$ & 1000 ppm ديابت+استات سرب & A \\
\hline$. / 1 \Delta \pm . / . \Delta$ & $\cdot / M I \pm \cdot / \cdot A$ & $. / g Y \pm . / N T$ & $<\cdot 1 . \Delta$ & 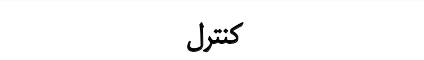 & كروه \\
\hline
\end{tabular}

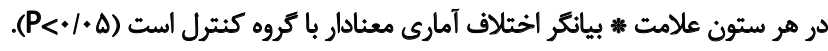



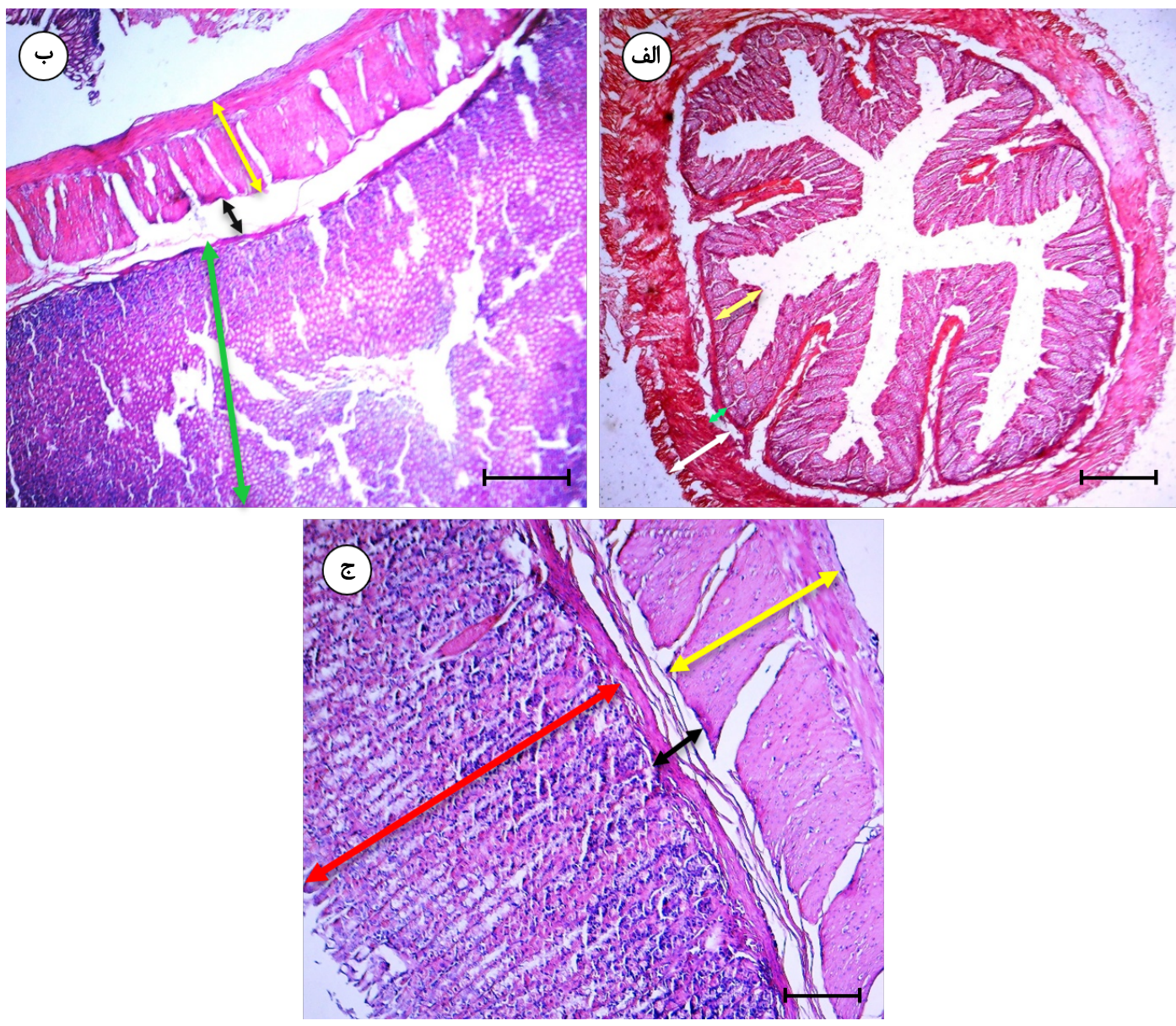

sins

تصوير ا. ميكروفتوكراف نورى تغييرات كمى هيستومورفومترى بافت رودهى موش صحرايى

تصوير الف: ميكروفتوكراف بافت كولون در كروه كنترل

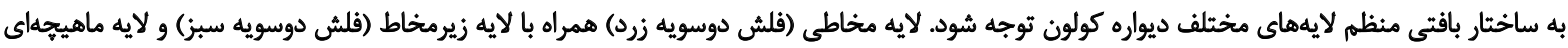
(فلش دوسويه سفيد) (رنكآميزى هماتوكسيلين و أثوزيني: Bar:

تصوير ب: ميكروفتوكراف بافت كولون در گروه ديابتي

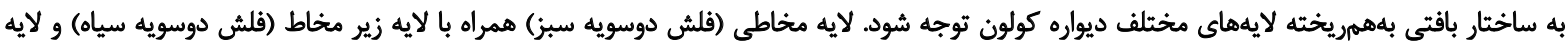

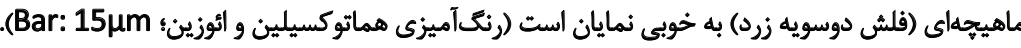

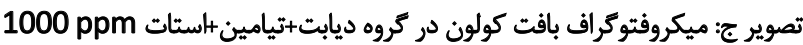

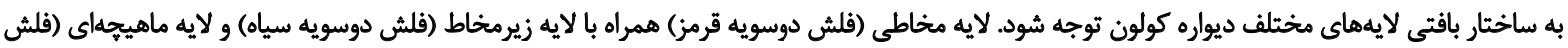

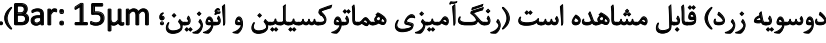

مى تواند ناشى از اختلال در ترشح انسولين باشد.

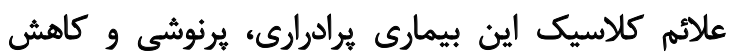

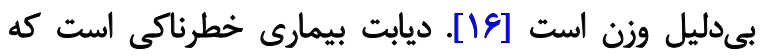

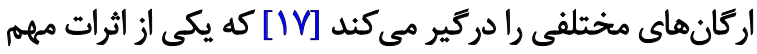

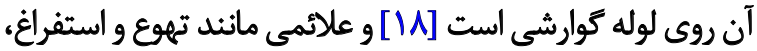

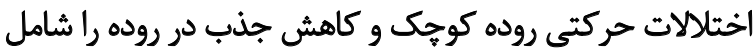

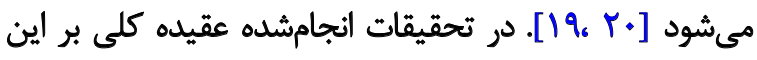

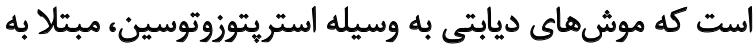
هيبرفارى و هييريلازى در روده شدند.
اين اثرات جها به صورت افزايش و جها به به صورت كاهش،

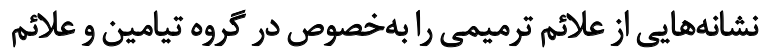

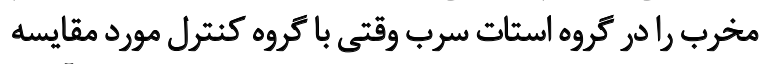

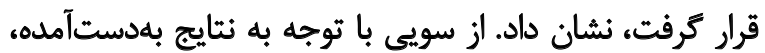

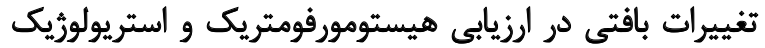

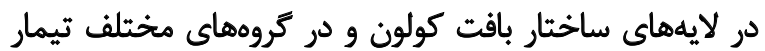

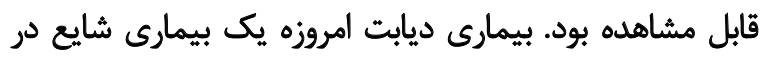

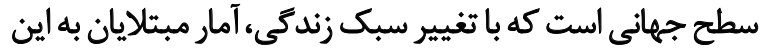

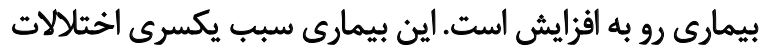

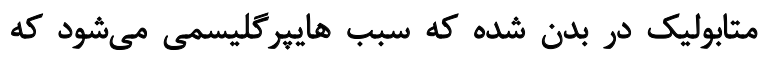


كند، به طورى كه ضخامت لايه عضلاتى و طول يرزها در دري

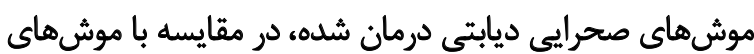

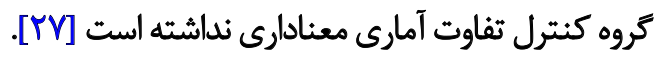
در مطالعه ما تزريق تيامين+استات دويست سبب كاهش

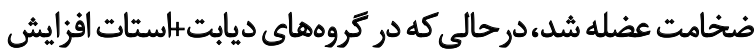
ضخامت اين لايه مشهود بود.

برخى مطالعات تغييرات تروفيك روده كوجى راء را ناشى از

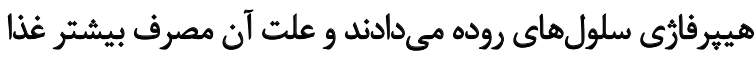

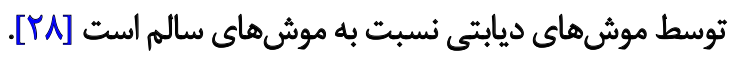
در ديابت به دليل ورود بيشتر غذا به معده شاهد حركات و

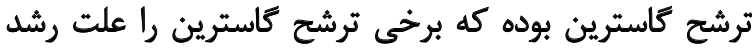

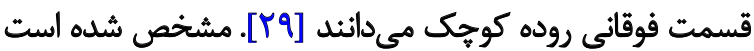

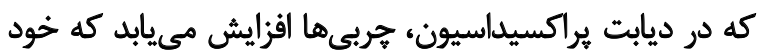

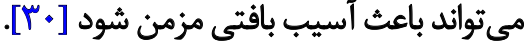
جهت بررسى ساختار ديواره بافت كولون، علاوه بر رهيافت

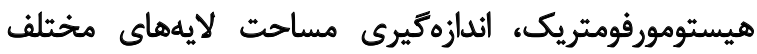

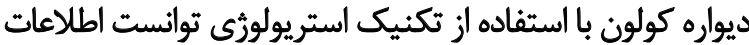
فزونترى در مطالعه حاضر در مورد اين ساختار ارائه دهد. استريولوزى به شاخهاى از علم ريختشناسى اطلاق مى إسود

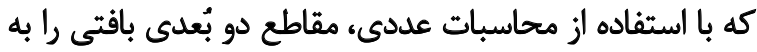

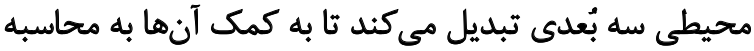

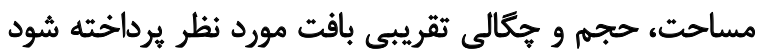

با توجه به نتايج مطالعه بيشرو، مي توان جنين بيان داشت

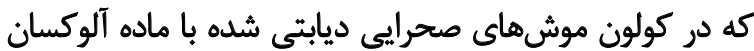

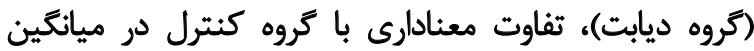

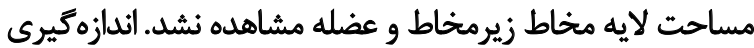

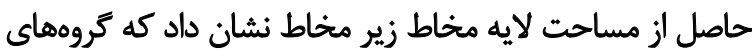

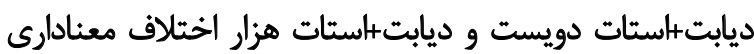

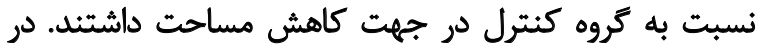

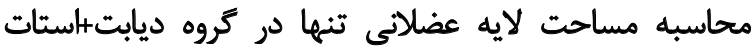
دويست افزايش مساحت نسبت به كروه كنترل ديده شدا ديد.

يافته سطحسنجى از بررسى استريولورثى نشان داد كه در كروه

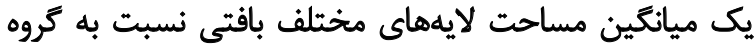

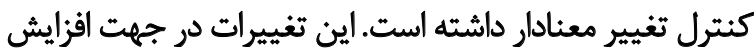

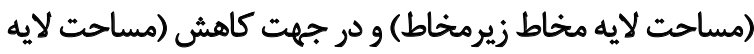

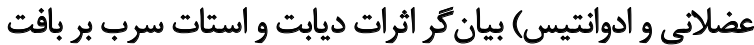

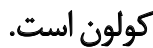

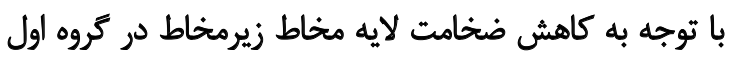

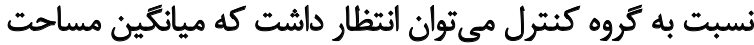
اين لايه نيز به تبع كاهش ضخامت، كاهش يابد ونتايج بهدست آمده.
نتايج مطالعه حاضر نشاندهندهى كاهش معنادار ضخامت

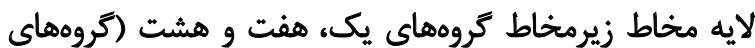

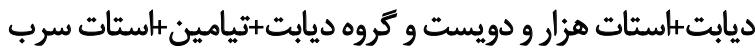

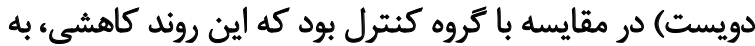

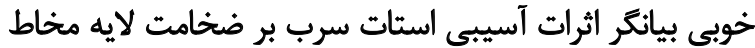

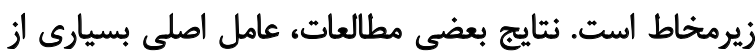

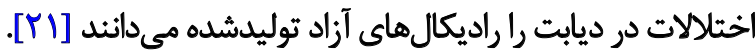

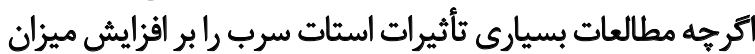

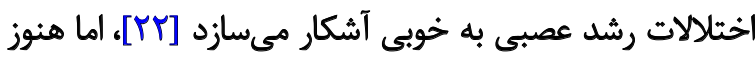

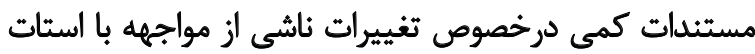

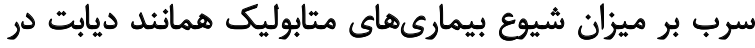

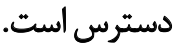
در مطالعات انجامشده روى اثرات سرب و مكانيسمهاى دائ

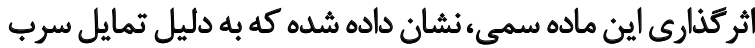

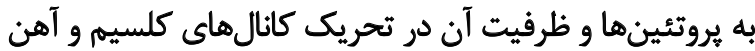

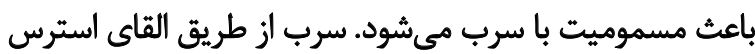

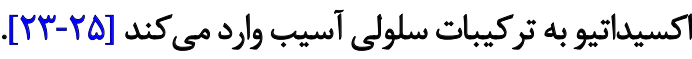
اززطرفىنتايجحاصل إزمقايسهكروكنترلياكروةتيمارديابت+تيامين

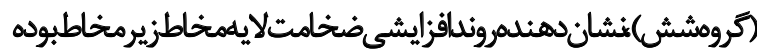

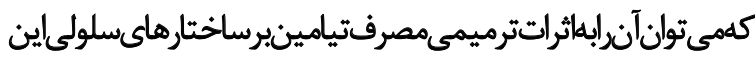
لايه به دنبال ديابت القايي نسبت دادئريت

با بررسى نتايج حاصل از مطالعهاى كه توسط درايدو همكاران

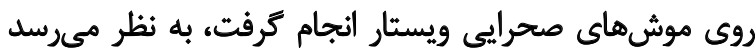

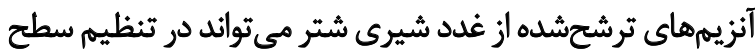

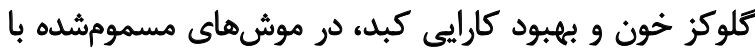

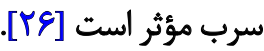
يافتهها حاكى از آن است كه ضخامت لايه عضلانى دستخوش تغييرات كاهشى در گروه ينج (ديابت) در مقايسه با باتروه كنترل

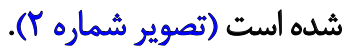
از سويى در مطالعهاى ديكر كه توسط نواك و همكاران صورت

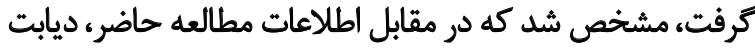

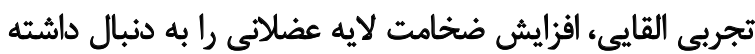

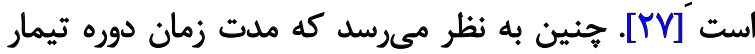

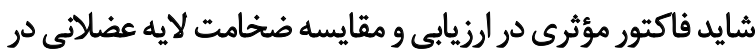

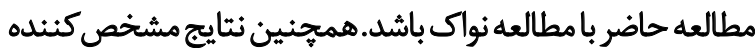

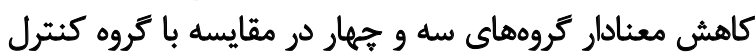

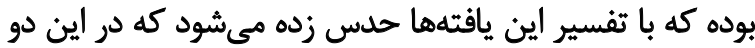

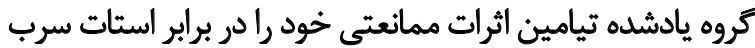

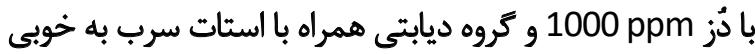
اعمال نكرده است (تصوير شماره ب). در نتايج حاصل از مطالعه كريمىيور و همكاران مشخص شد كه يودر زنجبيل توانسته است از اثرات ديابت بر روده جلوكيرى مئري شئ 
همجينين از معاونت محترم يثروهشي دانشكده و دانشكاه شهركرد

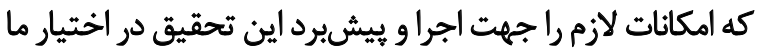
قرار دادند و مارا يارى كردند، تشكر و وقدر واتئي كنيند.

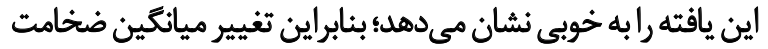

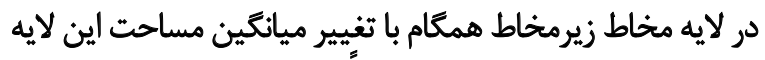
بوده و همخوانى اين دو فاكتور كاملاً مشهود استير ميانين

نتايج مطالعه ديكرى نشان داد كه ديابت باعث افزايش محيطى دئى

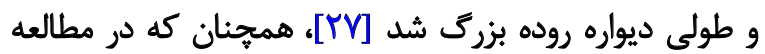

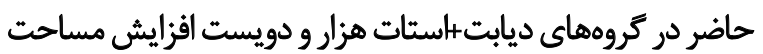

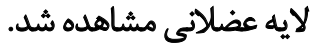

$$
\text { s.jus }
$$

جنان مى توان نتيجه كرفت كه با توجه به القاى ديابت تجربى تئى تئري

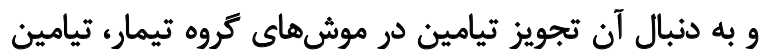

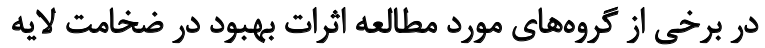

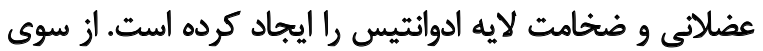

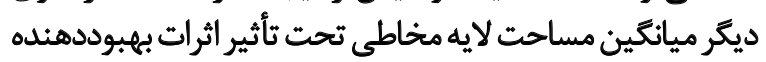
تيامين نبود. مالاحظات اخلاقى

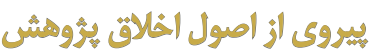

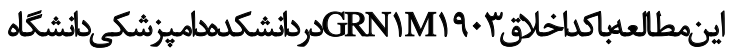

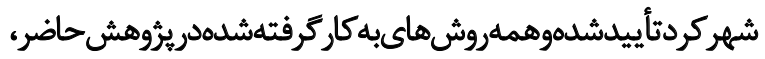

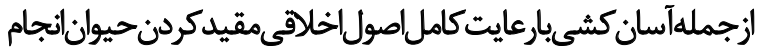
يذيرفت.

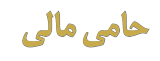

معاونتهاى محترم ثرؤششى دانشكده داميزشكى و دانشعاه

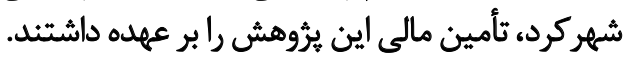

\section{مشار كت نويسندكان}

مفهومسازى: رحمتالله فتاحيان و سورن نورايي؛ تحقيق

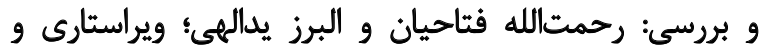

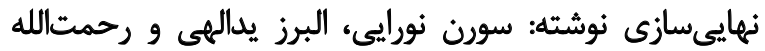

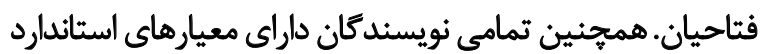

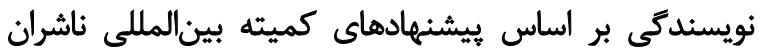
مجلات يزشكى (ICMJE) بودند.

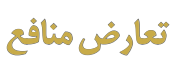

طبق نظر نويسندكان هيجگونه تضاد منافعى در يروهش حاضر وجود ندارد.

$$
\text { ش ش }
$$

نويسندكان مقاله حاضر، بر خود لازم مي دانئ كه از كارشناسان

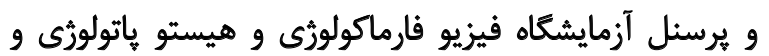




\section{Reference}

[1] Kassab E, McFarlane SI, Sowers JR. Vascular complications in diabetes and their prevention. Vasc Med. 2001; 6(4):249-55. [DOI:10.1177/1358 836X0100600409] [PMID]

[2] Mosayebi G, Ghaznavirad E, Fani E, Moazeni S. [Frequency of Human Leukocyte Antigens (HLA) class-I and II in arakian patients with insulin dependent diabetes mellitus (Persian)]. J Arak Univ Med Sci. 2006; 8(3):42-9. https://www.sid.ir/en/journal/ViewPaper.aspx?id=46344

[3] Forouhi NG, Wareham NJ. Epidemiology of diabetes. Medicine. 2019; 47(1):22-7. [DOI:10.1016/j.mpmed.2018.10.004]

[4] Hayashi T, Nozawa M, Sohmiya K, Toko H, Nakao M, Okabe M, et al. Efficacy of pancreatic transplantation on cardiovascular alterations in diabetic rats: An ultrastructural and immunohistochemical study. Transplant Proc. 1998; 30(2):335-8. [DOI:10.1016/S0041-1345(97)01295-5]

[5] Camilleri MJE, Clinics M. Gastrointestinal problems in diabetes Endocrinol Metab Clin. 1996; 25(2):361-78. [DOI:10.1016/S08898529(05)70328-5]

[6] Nathan D, Genuth S, Lachin J, Cleary P, Crofford O, Davis M, et al. The effect of intensive treatment of diabetes on the development and progression of long-term complications in insulin-dependent diabetes mellitus. N Engl J Med. 1993; 329(14):977-86. [DOI:10.1056/ NEJM199309303291401] [PMID]

[7] Musso G, Gambino R, Cassader M. Obesity, diabetes, and gut microbiota: The hygiene hypothesis expanded? Diabetes care. 2010; 33(10):2277-84. [DOI:10.2337/dc10-0556] [PMID] [PMCID]

[8] Amiri A, Fatahian Dehkordi RA, Heidarnejad MS, Jafarian Dehkordi M. Effect of the zinc oxide nanoparticles and thiamine for the management of diabetes in alloxan-induced mice: A stereological and biochemical study. Biol Trace Elem Res. 2018; 181(2):258-64. [DOI:10.1007/s12011017-1035-x] [PMID]

[9] Rindi G, Laforenza U. Thiamine intestinal transport and related issues: Recent aspects. Proc Soc Exp Biol Med. 2000; 224(4):246-55. [DOI:10.1111/j.1525-1373.2000.22428.x] [PMID]

[10] Quoc Luong KV, Nguyen LTH. The impact of thiamine treatment in the diabetes mellitus. J Clin Med Res. 2012; 4(3):153-60. [DOI:10.4021/joc$\mathrm{mr} 890 \mathrm{w}]$

[11] Sugiura S. When Was B1, the first Vitamin, discovered?: An alternative perspective. J Nutr Sci Vitaminol. 2016; 62(5):372-4. [DOI:10.3177/ jnsv.62.372] [PMID]

[12] Baht RV, Moy GG. Monitoring and assessment of dietary exposure to chemical contaminants. World Health Stat Q. 1997; 50(1-2):132-49. [PMID]

[13] Pitot HC. The molecular biology of carcinogenesis. Cancer. 1993; 72(S3):963-70. [DOI:10.1002/1097-0142(19930801)72:3+3.0.CO;2-H]

[14] Sharma R, Mogra S. Lead as a developmental toxicant: A review. Int J Pharm Sci Res. 2014; 5(3):636-42. [DOI:10.13040/IJPSR.0975-8232.5(3).636-42]

[15] Leff T, Stemmer P, Tyrrell J, Jog R. Diabetes and exposure to environmental lead (Pb). Toxics. 2018; 6(3):54. [DOI:10.3390/toxics6030054] [PMID] [PMCID]

[16] Laakso M. Hyperglycemia and cardiovascular disease in type 2 diabetes. Diabetes. 1999; 48(5):937-42. [DOI:10.2337/diabetes.48.5.937] [PMID]
[17] Chaikomin R, Rayner CK, Jones KL, Horowitz M. Upper gastrointestinal function and glycemic control in diabetes mellitus. World J Gastroenterol. 2006; 12(35):5611-21. [DOI:10.3748/wjg.v12.i35.5611] [PMID] [PMCID]

[18] Anitha M, Gondha C, Sutliff R, Parsadanian A, Mwangi S, Sitaraman $S V$, et al. GDNF rescues hyperglycemia-induced diabetic enteric neuropathy through activation of the PI3K/Akt pathway. J Clin Invest. 2006; 116(2):344-56. [DOI:10.1172/JCI26295] [PMID] [PMCID]

[19] Maleki D, Locke 3rd GR, Camilleri M, Zinsmeister AR, Yawn BP, Leibson $C$, et al. Gastrointestinal tract symptoms among persons with diabetes mellitus in the community. Arch Gen Intern Med. 2000; 160(18):2808-16. [DOI:10.1001/archinte.160.18.2808] [PMID]

[20] Rayner CK, Horowitz M. Gastrointestinal motility and glycemic control in diabetes: The chicken and the egg revisited? J Clin Invest. 2006; 116(2):299-302. [DOI:10.1172/JCI27758] [PMID] [PMCID]

[21] Nourooz-Zadeh J, Rahimi A, Tajaddini-Sarmadi J, Tritschler H, Rosen $P$ Halliwell B, et al. Relationships between plasma measures of oxidative stress and metabolic control in NIDDM. Diabetologia. 1997; 40(6):64753. [DOI:10.1007/s001250050729] [PMID]

[22] Searle AK, Baghurst PA, Van Hooff M, Sawyer MG, Sim MR, Galletly C et al. Tracing the long-term legacy of childhood lead exposure: A review of three decades of the port Pirie cohort study. Neurotoxicology. 2014 43:46-56. [DOI:10.1016/j.neuro.2014.04.004] [PMID]

[23] Ayoubi A, Vaizadeh R, Omidi A, Abolfazli MD. Protective effects of vitamin $C$ (ascorbic acid) in lead acetate exposed diabetic male rats: Evaluation of blood biochemical parameters and testicular histopathology. Istanbul Univ Vet Fak Derg. 2014; 41(1):84-91. [DOI:10.16988/iuvfd.2015.90715]

[24] Hakim ZS, Patel BK, Goyal RK. Effects of chronic ramipril treatment in streptozotocin-induced diabetic rats. Indian J Physiol Pharmacol. 1997; 41(4):353-60. [PMID]

[25] Jenkinson KM, Reid JJ. Effect of diabetes on relaxations to nonadrenergic, non-cholinergic nerve stimulation in longitudinal muscle of the rat gastric fundus. Br J Pharmacol. 1995; 116(1):1551-6. [DOI:10.1111/j.1476-5381.1995.tb16372.x] [PMID] [PMCID]

[26] Draid MM, Bushwereb MO, Ramadan SG. Lead acetate toxicity on glucose level and liver enzymes amelioratwd by camel's milk in wistar albino rat. Int J Basic Clin Pharmacol. 2016; 5(3):1125-30. [DOI:10.18203/23192003.ijbcp20161580]

[27] Nowak TV, Chey WW, Chang T-M, Weisbruch JP, Fouquet G. Effect of streptozotocin-induced diabetes mellitus on release of vasoac tive intestinal polypeptide from rodent small intestine. Dig Dis Sci. 1995;40(4):828-36. [DOI:10.1007/BF02064987] [PMID]

[28] Jervis EL, Levin RJ. Anatomic adaptation of the alimentary tract of the rat to the hyperphagia of chronic alloxan-diabetes. Nature. 1966; 210(5034):391-3. [DOI:10.1038/210391a0] [PMID]

[29] Ekundayo AA, Lee CY, Goodlad RA. Gastrin and the growth of the gastrointestinal tract. Gut. 1995; 36(2):203-8. [DOI:10.1136/gut.36.2.203] [PMID] [PMCID]

[30] Afshari AT, Shirpoor A, Farshid A, Saadatian R, Rasmi Y, Saboory E, et al. The effect of ginger on diabetic nephropathy, plasma antioxidant capacity and lipid peroxidation in rats. Food Chem. 2007; 101(1):148-53. [DOI:10.1016/j.foodchem.2006.01.013]

[31] Fatahian Dehkordi RA, Habibi A. Stereological and electron microscopic study on the internal organization of the urinary secretory units in three species of birds. J Appl Animal Res. 2015; 43(1):118-24. [DOI:10 .1080/09712119.2014.928625] 
This Page Intentionally Left Blank 